\title{
Light-Induced Subpicosecond Lattice Symmetry Switch in $\mathrm{MoTe}_{2}$
}

\author{
M. Y. Zhang, ${ }^{1}$ Z. X. Wang, ${ }^{1}$ Y. N. Li, ${ }^{1}$ L. Y. Shi, ${ }^{1}$ D. Wu, ${ }^{1}$ T. Lin, ${ }^{1}$ S. J. Zhang, ${ }^{1}$ Y. Q. Liu, ${ }^{1}$ \\ Q. M. Liu, ${ }^{1}$ J. Wang, ${ }^{1,2}$ T. Dong, ${ }^{1, *}$ and N. L. Wang ${ }^{1,2, \dagger}$ \\ ${ }^{1}$ International Center for Quantum Materials, School of Physics, Peking University, Beijing 100871, China \\ ${ }^{2}$ Collaborative Innovation Center of Quantum Matter, Beijing, China
}

(Received 26 September 2018; published 22 May 2019)

\begin{abstract}
The recent development of ultrashort laser pulses allows for optical control of structural and electronic properties of complex quantum materials. The layered transition-metal dichalcogenide $\mathrm{MoTe}_{2}$, which can crystallize into several different structures with distinct topological and electronic properties, provides possibilities to control or switch between different phases. In this study, we report a photoinduced subpicosecond structural transition between the type-II Weyl semimetal phase and normal-semimetal phase in bulk crystalline $\mathrm{MoTe}_{2}$ by using ultrafast pump-probe and time-resolved second-harmonic-generation spectroscopy. The phase transition is most clearly characterized by the dramatic change of the shear oscillation mode and the intensity loss of second-harmonic generation. This work opens up new possibilities for ultrafast manipulation of the topological properties of solids, enabling potentially practical applications for a topological switch device with ultrafast excitations.
\end{abstract}

\section{INTRODUCTION}

Layered transition-metal dichalcogenide (TMD) $M X_{2}$ [where $M$ is a transition metal ( $\mathrm{Ta}, \mathrm{Nb}, \mathrm{Mo}$, and $\mathrm{W})$, and $X$ is a chalcogen ( $\mathrm{S}, \mathrm{Se}$, and $\mathrm{Te})]$ represents one of the most interesting material groups that shows rich physical phenomena such as charge-density wave, superconductivity, quantum spin Hall effect, topological nontrivial state, unsaturated extremely large magnetoresistivity, etc. Among them, $\mathrm{MoTe}_{2}$ has recently attracted particular attention because it hosts type-II topological Weyl fermions in a noncentrosymmetric octahedral $T_{d}$ structural phase [1-3], which allows for fundamental studies of intriguing topological physics. Actually, $\mathrm{MoTe}_{2}$ can crystallize into several different structures with different properties, for example, the trigonal prismatic coordinated hexagonal $2 \mathrm{H}$ phase (space group $\mathrm{Pb}_{3} / \mathrm{mmc}$ ), distorted octahedral coordinated monoclinic $1 T^{\prime}$ (space group $P 2_{1} / \mathrm{m}$ ), and orthorhombic $T_{d}$ (space group $P m n 2_{1}$ ) phases [4-6]. The $2 H$ phase has a central symmetric structure and is semiconducting, but the $1 T^{\prime}$ and $T_{d}$ phases show semimetallic nature. The $1 T^{\prime}-\mathrm{MoTe}_{2}$ phase is centrosymmetric and can be transformed into the $T_{d}-\mathrm{MoTe}_{2}$ phase with broken

\footnotetext{
taodong@pku.edu.cn

†nlwang@pku.edu.cn
}

Published by the American Physical Society under the terms of the Creative Commons Attribution 4.0 International license. Further distribution of this work must maintain attribution to the author(s) and the published article's title, journal citation, and DOI. inversion symmetry by cooling the compound below approximately $250 \mathrm{~K}[4,5]$. $T_{d}-\mathrm{MoTe}_{2}$ is also found to be superconducting with $T_{c}=0.1 \mathrm{~K}$, thus, serving as a promising candidate for the topological superconductor [7-9]. Since the energy difference between different phases is small in $\mathrm{MoTe}_{2}$ [10], the system provides a good possibility to manipulate or engineer controlled phase transitions.

The lattice structures and symmetries of materials are usually probed and determined by elastic x-ray, neutron, and electron diffractions or inelastic Raman spectroscopy. With the development of advanced femtosecond laser techniques, ultrafast coherent phonon spectroscopy based on the pump-probe technique and second-harmonicgeneration (SHG) spectroscopy provide alternative routes to resolve lattice symmetry [11-17]. Such optical probes are particularly effective for detecting structural change associated with inversion symmetry breaking. When the inversion symmetry is broken, parity is not a good quantum number, giving rise to a mixture of even and odd states. The sudden lattice symmetry change would lead to a variation in $A_{1}$ Raman active phonon modes, which can be tracked by the ultrafast coherent phonon spectroscopy through the displacive excitation of coherent phonons or stimulated Raman-scattering process [18,19]. SHG spectroscopy is extremely sensitive to inversion symmetry because the leading-order contribution to the SHG signal is nonzero only when the inversion symmetry is broken, e.g., at the interfaces or in bulk noncentrosymmetric crystalline. More importantly, the employment of such optical probes allows for time-resolved measurement after ultrafast photoexcitations. Up to now, the experimental realization of 
a switch between different structural phase transitions in $\mathrm{MoTe}_{2}$ was mainly achieved via application of strain, doping, or static electric field on few-layer samples [20-22]. Ultrashort laser pulses provide a new route to manipulate the structural and electrical properties of a quantum material. When the electric field of the pumping pulse is comparable to the potential gradients of atoms, the intense pulse can change the landscape of free energy and induce a phase transition. There are many good examples of the ultrafast photoinduced transitions such as the insulatorto-metal transition in $\mathrm{VO}_{2}[12,23]$, the lattice distortions in manganites [24], and the charge-density-wave insulator to metal transition in quasi-one-dimensional $\mathrm{K}_{0.3} \mathrm{MoO}_{3}$ $[11,25]$. A recent study indicated that strong laser radiation can induce a nonreversal structural phase transition between the $2 \mathrm{H}$ and $1 \mathrm{~T}$ phases in $\mathrm{MoTe}_{2}$ [26]. Here we report an ultrafast photoinduced subpicosecond structural transition between the type-II Weyl semimetal $T_{d}$ phase and normalsemimetal $1 T^{\prime}$ phase in bulk crystalline $\mathrm{MoTe}_{2}$. The onset and degree of the phase transition are determined by transient photoinduced coherent phonon spectroscopy and timeresolved SHG separately.

\section{SAMPLE CHARACTERIZATION}

The $\mathrm{MoTe}_{2}$ single crystals are grown by vapor-transport method using iodine as the transport agent, similar to the method reported in earlier work [9]. The high-temperature centrosymmetric monoclinic $1 T^{\prime}$ structure and lowtemperature noncentrosymmetric $T_{d}$ structure are shown in the Figs. 1(a) and 1(b), respectively. The structural difference between the two phases is small, only by a $4^{\circ}$ tilting angle from the stacking direction ( $c$ axis). The typeII Weyl semimetal characteristics in the $T_{d}$ phase are identified by angle-resolved-photoemission-spectroscopy and scanning-tunneling-spectroscopy studies [27,28].

We perform temperature-dependent resistivity and optical SHG measurements on the crystals. The resistivity measurement is measured by a standard four-probe method on a Quantum Design physical properties measurement
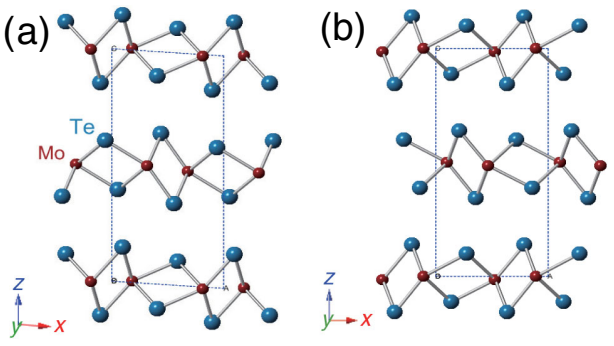

(d)

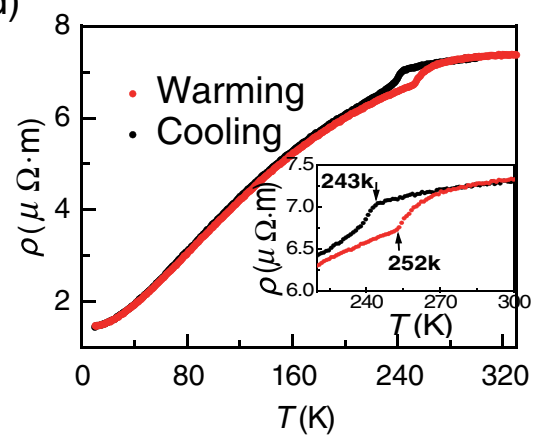

(f)

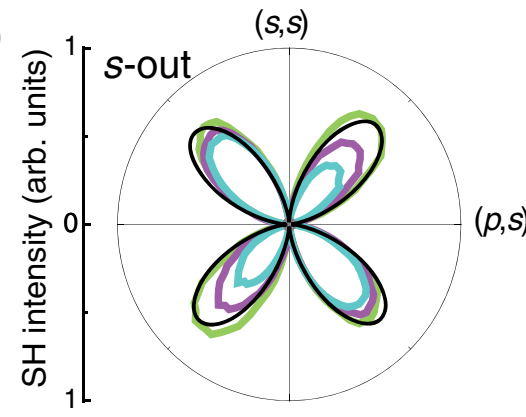

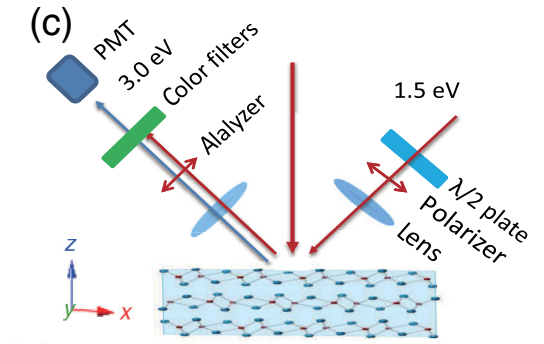

(e)

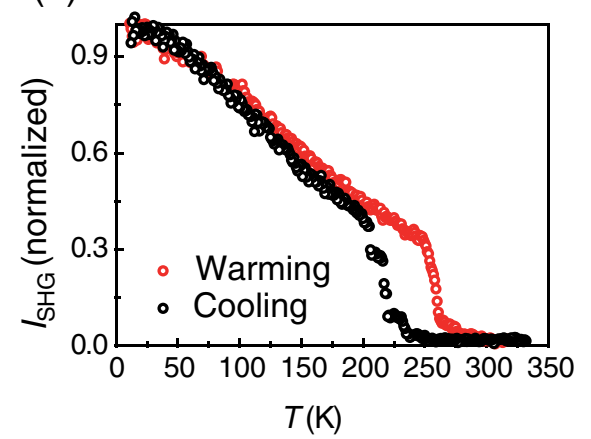

(g)

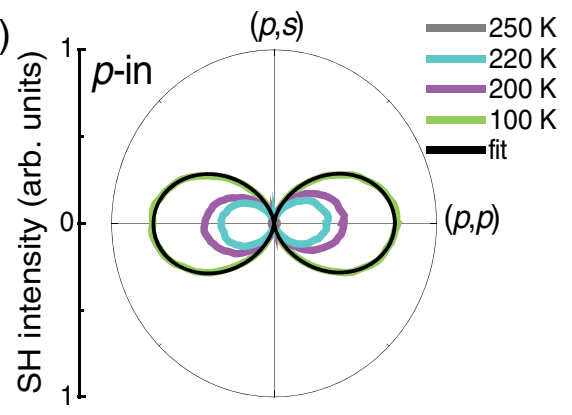

FIG. 1. Sample structure and characterization. Crystal structures of $\mathrm{MoTe}_{2}$ in the (a) $1 T^{\prime}$ and (b) $T_{d}$ phases. Beam path in the SHG experiment is shown in (c). (d) $T$-dependent resistivity. (e) Optical SHG intensity measured with an incident photon energy of $800 \mathrm{~nm}$. Both $\rho(T)$ and the SHG measurement show a temperature-induced first-order phase transition. SHG characteristics (f) for the $s$-polarized $\mathrm{SH}$ photons while the incidence polarizer is rotated and $(\mathrm{g})$ for the incidence $p$ polarized while the SH analyzer is rotated. 
system. The SHG is measured with a Ti:sapphire amplified laser of $800-\mathrm{nm}$ pulses with $100-\mathrm{fs}$ duration at $1-\mathrm{kHz}$ repetition rate focusing on the $a b(x y)$ plane of the sample ( 0.1 to $0.8 \mathrm{~mW}$ on a $110-\mu \mathrm{m}$ spot) in reflection geometry at a $45^{\circ}$ angle of incidence. A schematic diagram of the optical path arrangement is shown in Fig. 1(c). The $p(s)$ polarization of the incidence is in the $a c$ plane ( $x z$ plane) (along the $b$ axis) of the sample crystal. The incident polarization is controlled by a half wave plate, and the polarization of the reflection SHG is analyzed by a Glan laser prism. Figure 1(d) shows the temperature-dependent resistivity of the crystal. The presence of significant hysteresis in the transition temperatures between cooling down (about $240 \mathrm{~K}$ ) and warming up (about $250 \mathrm{~K}$ ) [inset of Fig. 1(d)] yields evidence for the first-order phase transition at $T_{V}$. Figure 1(e) shows the optical SHG intensity at a particular measurement configuration of $p$-polarized incidence and $p$-polarized second-harmonic (SH) photons. The signal is absent at high temperature but increases suddenly below the phase transition, yielding evidence for the inversion symmetry breaking in the $T_{d}$ phase. The signal also shows a clear hysteresis across $T_{V}$ with cooling down and warming up the sample, further supporting the first-order phase transition.

Figures $1(\mathrm{f})$ and $1(\mathrm{~g})$ show the $\mathrm{SH}$ polarization patterns in $\mathrm{MoTe}_{2}$ for two different measurement configurations, with a fixed $s$-polarized incidence-rotatable $\mathrm{SH}$ analyzer and a fixed $p$-polarized $\mathrm{SH}$ photon-rotatable incidence polarizer, respectively. For Fig. 1(f), the incident electric field $\hat{e}_{\omega}{ }^{2}=\left[E_{x}^{2}, E_{y}^{2}, E_{z}^{2}, 2 E_{y} E_{z}, 2 E_{z} E_{x}, 2 E_{x} E_{y}\right]=\left[\frac{1}{2} \cos ^{2} \theta, \sin ^{2} \theta\right.$, $\left.\frac{1}{2} \cos ^{2} \theta,-\sqrt{2} \sin \theta \cos \theta,-\cos ^{2} \theta, \sqrt{2} \sin \theta \cos \theta\right](\theta$ is the angle the incidence polarizer rotates from $p$ polarization) generates a second-harmonic signal along the $\hat{e}_{2 \omega}=[0,1,0]$ for $s$ polarization. The generated SHG intensity $I_{\mathrm{SHG}}$ can be expressed as $I_{\mathrm{SHG}} \propto\left|\hat{e}_{2 \omega} \cdot \mathbf{d}: \hat{e}_{\omega}^{2}\right|^{2}$, where $\mathbf{d}$ is the contracted notation of the second-order susceptibility tensor. d is a $3 \times 6$ second-rank tensor, and the nonzero tensor elements for the $m m 2$ point group are $d_{15}, d_{24}, d_{31}, d_{32}$, and $d_{33}$. So, we can easily lead to $I_{s-\text { out }} \propto d_{24}^{2} \sin ^{2} 2 \theta$. It is used to fit the polarization pattern in Fig. 1(f) very well. Similarly, when $\theta=0$, the incident electric field is $\hat{e}_{\omega}{ }^{2}=$ $\left[E_{x}^{2}, E_{y}^{2}, E_{z}^{2}, 2 E_{y} E_{z}, 2 E_{z} E_{x}, 2 E_{x} E_{y}\right]=\left[\frac{1}{2}, 0, \frac{1}{2}, 0,-1,0\right]$. We fit the lobes in Fig. $1(\mathrm{~g})$ by equation $I_{p-\text { in }} \propto\left(-d_{15}+d_{31} /\right.$ $\left.2+d_{33} / 2\right)^{2} \cos ^{2} \varphi$, where the SH analyzer is rotated at an angle $\varphi$ from $p$ polarization. The polarization patterns can be well reproduced by the symmetry analysis based on the space group $P m n 2_{1}$ further proving the structure of the compound [21].

\section{ULTRAFAST COHERENT PHONON SPECTROSCOPY}

In order to monitor the ultrafast dynamics of the phase transition, the equilibrium technique can be extended to the time domain via a standard pump-probe configuration.
A Ti:sapphire amplified laser of 800-nm pulses with 35-fs duration at $1-\mathrm{kHz}$ repetition rate is utilized as the source of both the pump and probe beams. The pump beam is modulated by a chopper at a frequency of $333 \mathrm{~Hz}$ and polarized perpendicular to the probe beam ( $p$-polarized pump, $s$-polarized probe). We keep the pump fluence at about $100 \mu \mathrm{J} / \mathrm{cm}^{2}, 10$ times stronger than the probe pulses. Figures 2(a) and 2(b) show the photoinduced reflectivity change $\Delta R / R$ as a function of time delay measured from 4 to $300 \mathrm{~K}$ (the warming process) and from 300 to $4 \mathrm{~K}$ (the cooling process) separately. The decay dynamics contains two processes [29,30]: a fast component $\left(\tau_{1}\right)$ about $0.3 \mathrm{ps}$ and an almost constant or very slow component $\left(\tau_{2}\right)$ about hundreds of picoseconds. The coherent vibrational dynamics is obtained after subtracting the two decay processes. The coherent vibrational dynamics contains the oscillation signals originating from the low-energy $A_{1}$ optical phonons [18]. In principle, the reflectivity changes induced by coherent phonons arise from the modulation of the optical susceptibility at the phonon frequency. The dielectric susceptibility can be expressed by displacement coordinate $Q(t)$ around equilibrium as $\chi=\chi_{0}+\{[(\partial \chi) /(\partial Q)] * Q(t)\}$, where the second-rank tensor $\left(\partial \chi^{(1)}\right) /(\partial Q)$ is linked to the Raman tensor, and the third-rank tensor $\left(\partial \chi^{(2)}\right) /(\partial Q)$ is related to the hyper-Raman tensor [17]. It can be seen clearly in the time-domain spectrum that a long-period optical coherent phonon suddenly disappears above the structural phase-transition temperature.

Figures 2(c) and 2(d), respectively, show a Fourier transform of these oscillations of Figs. 2(a) and 2(b) after subtracting the two-exponential-decay components of the form $\Delta R / R=A_{0}+A_{1} \quad \exp \left(-t / \tau_{1}\right)+A_{2} \quad \exp \left(-t / \tau_{2}\right)$, where $A_{i}$ and $\tau_{i}(i=1,2)$ are the fit parameters that represent the amplitude and recovery rate of the dynamics, respectively. Six distinct peaks are observed in the Fourier spectrum at $0.42,2.3,3.4,3.89,5.0$, and $7.77 \mathrm{THz}$ (approximately 14, 77, 113, 130, 167, and $259 \mathrm{~cm}^{-1}$ ) at the low temperature. We found that all these phonons have been detected in the Raman spectrum as $A_{1}$ modes [31-33]. The $0.42-\mathrm{THz}$ phonon, which appears around $240 \mathrm{~K}$ during the cooling process [Fig. 2(d)] and disappears around $250 \mathrm{~K}$ during the warming process, has much stronger intensity [Fig. 2(c)]. It shows the same hysteresis as observed in the temperature-dependent resistivity and SHG signals. The same hysteresis has also been reported in the Raman measurement [31,32]. Based on the symmetry analysis and calculation results reported before, the $0.42-\mathrm{THz}$ phonon is a shear mode between layers and is Raman active in the $T_{d}$ phase and inactive in the $1 T^{\prime}$ phase (since the zone-center lattice vibrations have odd parity in the inversion-symmetric structure in the $1 T^{\prime}$ phase) [32]. So, we can take the $0.42-\mathrm{THz}$ shear mode as an indication of the change of the structural transition in this compound.

Figure 2(e) shows the temperature evolution of the amplitude of the phonon at $0.42 \mathrm{THz}$ together with other 

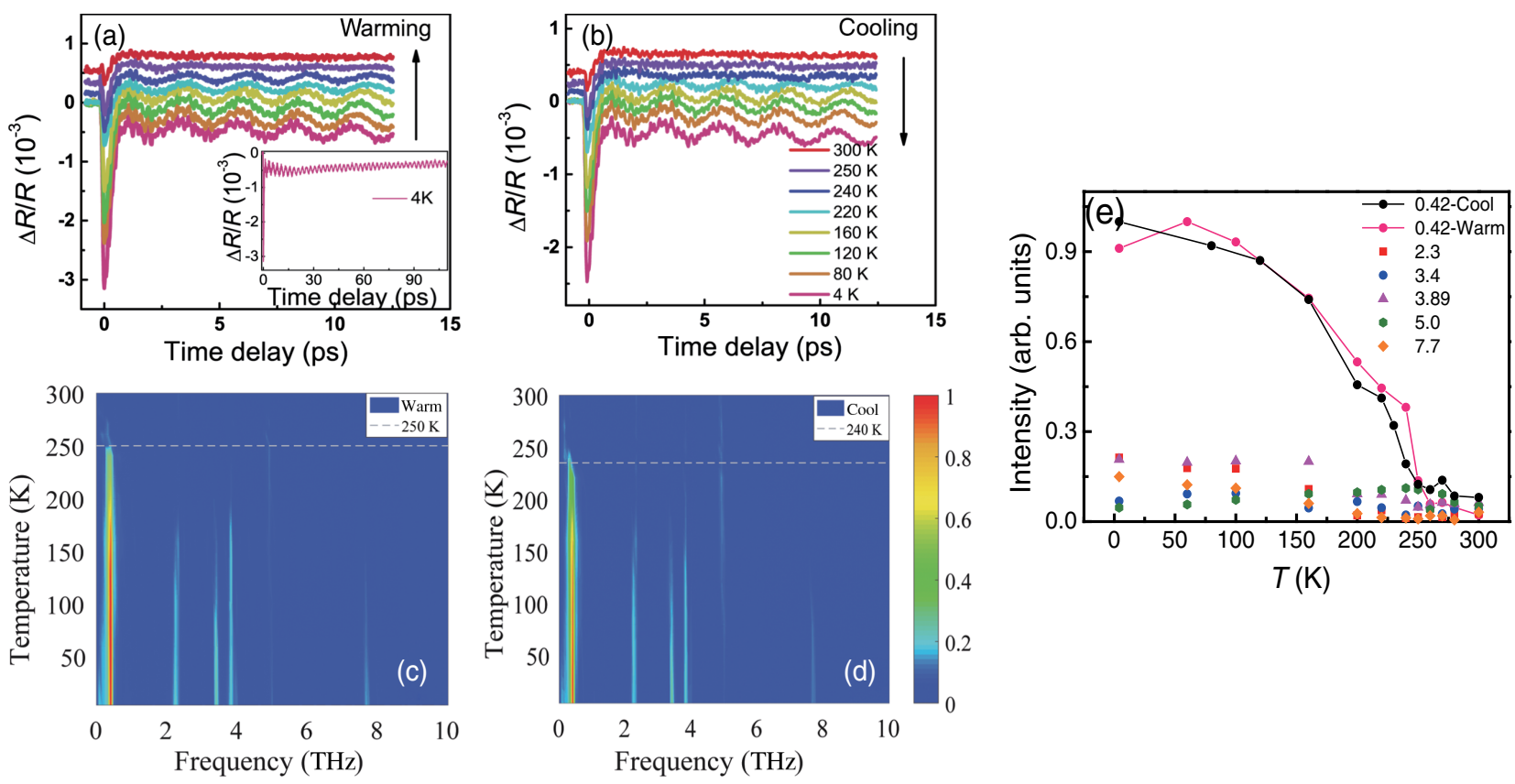

FIG. 2. The temperature-dependent coherent vibrational dynamics. The transient-pump-induced reflectivity at $800 \mathrm{~nm}$ (a) from 4 to $300 \mathrm{~K}$ (warming process) and (b) from 300 to $4 \mathrm{~K}$ (the cooling process). Above $240 \mathrm{~K}$, all the curves are vertically shifted for clarity. Inset of (a): Pump-induced reflectivity change at a longer time-delay scale at $4 \mathrm{~K}$. We can see that the coherent phonons last for a very long time delay. Color plots of the Fourier transformation of the transient reflectivity after subtracting the background: (c) the warming process and (d) the cooling process at selected temperatures. (e) The temperature dependence of the amplitudes of different phonons during the warming process.

modes extracted from Fig. 2(c). In principle, the other $A_{1}$ phonon modes should also change at the phase transition. However, since the structural change is very subtle and the related modes at similar frequencies are also present in the $1 T^{\prime}$ phase based on calculations [31-33], their change cannot be well resolved. Additionally, the phonon intensity decreases as the temperature increases, as shown in Fig. 2(e). Because of the enhanced scattering from electrons at elevated temperature, some weak modes gradually disappear at temperatures even much lower than $250 \mathrm{~K}$, while the strong mode at $0.42 \mathrm{THz}$ remains but drops suddenly at Tv.

Having identified the shear mode as an indication of structural transition $[31,32,34]$, we now explore the fluence dependence of the pump-probe signal. Figure 3(a) presents the pump-induced reflectivity change of $\mathrm{MoTe}_{2}$ for different selected pump fluences at the low temperature $T=4 \mathrm{~K}$. The curves are vertically shifted for clarity. The Fourier transformations of the oscillations after subtracting the background are shown in Fig. 3(b). Under very low fluence, the signal is so small that only the strongest $0.42-\mathrm{THz}$ phonon can be detected. With increasing the fluence, the other five phonons show up, but the $0.42-\mathrm{THz}$ phonon tends to disappear near $5 \mathrm{~mJ} / \mathrm{cm}^{2}$. Figure 3(c) displays the fluence dependence of the maximum change of the reflectivity [peak intensity in Fig. 3(a)]. It is clearly seen that the maximum change saturates at high fluence. Figure 3(d) shows the fluence dependence of the amplitudes of different phonons. It can be seen that amplitudes of other phonons slightly increase with fluence. It satisfies the theory of displacive excitation of coherent phonons [18] that the intensity of the coherent phonon should increase with increasing fluence before reaching the damage threshold of a material [35]. But for the amplitude of the shearmode phonon at $0.42 \mathrm{THz}$, it increases linearly at small fluence and then decreases after reaching a maximum near $1.5 \mathrm{~mJ} / \mathrm{cm}^{2}$.

There could be two possible reasons for the intensity reduction of the $0.42-\mathrm{THz}$ phonon mode in the high fluence. One is a dephasing mechanism arising from oscillation damping and another is due to the symmetry change of the lattice structure. Since the coherent phonon manifests as an oscillation in the recorded time trace of transient reflectivity, we can roughly estimate the dephasing time from the damping of the oscillation. As shown in Fig. 3(a), the oscillation still lasts for a long time at the high pump fluence, suggesting an almost unchanged lifetime of the coherent phonon, which essentially excludes the dephasing mechanism. Then we need to consider the pump-induced structural symmetry change. The compound has a first-order transition at about $250 \mathrm{~K}$, and the $0.42-\mathrm{THz}$ phonon mode disappears in the high-temperature phase while other modes at similar frequencies remain [31]. A possible scenario is that the intensive pump can directly drive a structural change from the $T_{d}$ to $T^{\prime}$ phase. Photoinduced structural phase transitions have been found in some other compounds [24,36,37], but the mechanisms 

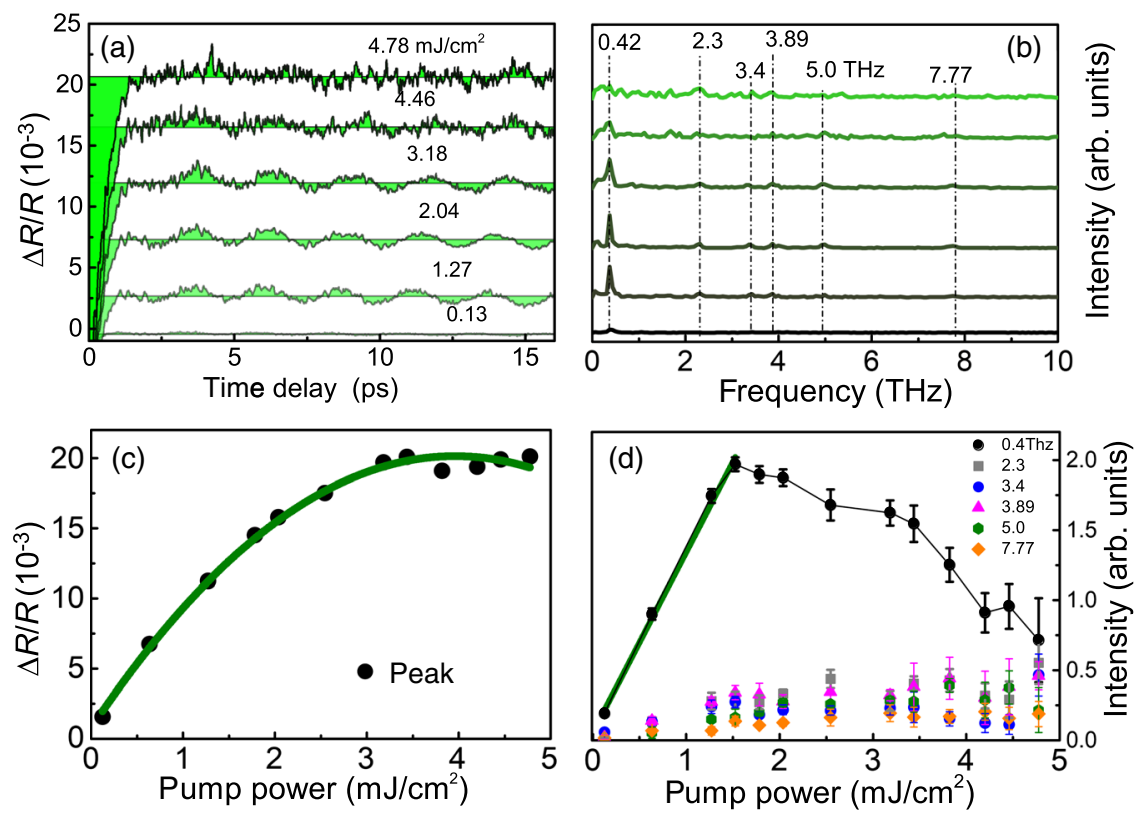

FIG. 3. The fluence-dependent coherent vibrational dynamics. (a) The transient-pump-induced reflectivity changes in MoTe 2 at $4 \mathrm{~K}$ with different excitation fluences. (b) The Fourier transformation of the transient reflectivity after subtracting the background in (a). (c) The fluence dependence of the maximum change of the reflectivity. The green curve is a guide to the eyes. (d) The fluence dependence of amplitudes of different phonons. The intensity increases linearly at small fluence.

of the transition remain debated. Another possibility is the thermal effect in the lattice temperature induced by the intense pump $[38,39]$. In the latter case, the expected temperature rise $\Delta T$ at temperature $\left(T_{0}\right)$ can be estimated by the equation $S \delta \rho / M \int_{T_{0}}^{T_{0}+\Delta T} C_{p}(T) d T=(1-R) F S$, where $S, \rho, M, R, F$, and $\delta$ are the excitation area, the mass density $\left(7.78 \mathrm{~g} / \mathrm{cm}^{3}\right)$, the molar mass $(351.1 \mathrm{~g} / \mathrm{mol})$, the reflectivity of $\mathrm{MoTe}_{2}$ (about 0.45 at $800 \mathrm{~nm}$ from our own measurement), the laser fluence, and the optical penetration depth $(\delta \approx 100 \mathrm{~nm})$, respectively. $C_{p}$ is the temperature-dependent thermal capacity. We estimate the values of $\Delta T$ to be approximately 120 and $300 \mathrm{~K}$ with excitation fluence about 1 and $5 \mathrm{~mJ} / \mathrm{cm}^{2}$, respectively, at $T_{0}=5 \mathrm{~K}$. It seems that the thermal effect can also explain the observation. However, it is hard to define the accurate temperature increase in ultrafast laser excitation since thermal diffusion is neglected in the equation. In the next section, we explain that the latter possibility is unlikely or can be essentially ruled out. As a matter of fact, the timescale of the phase transition appears to be more reliable evidence to distinguish between the thermal and nonthermal effects $[12,40]$.

\section{THE TIME-RESOLVED SECOND-HARMONIC GENERATION}

It is well known that the nonlinear susceptibility (especially second order) is very sensitive to the symmetry change of a lattice [15-17,41]. As we demonstrate in Fig. 1(d), the SH intensity in $\mathrm{MoTe}_{2}$ is nearly zero in the
$1 T^{\prime}$ phase while sharply enhanced in the $T_{d}$ phase where inversion symmetry is broken; hence, more valuable insight into pump-induced lattice symmetry change can be gained from the time-resolved second-harmonic generation (TR SHG) spectroscopy. We perform a near-infrared $(800 \mathrm{~nm})$ pump, SHG measurement on the sample as seen in Fig. 1(c). A Ti:sapphire amplified laser system of 800-nm laser pulses with 100 -fs duration at $1-\mathrm{kHz}$ repetition rate is used for the measurement. The spot sizes are about $150 \mu \mathrm{m}$ for the pump and $110 \mu \mathrm{m}$ for the probe in $45^{\circ}$ configuration. The pump is in normal incidence, and the scattering surface is in the ac plane of the compound. Earlier structural study based on the crystallographic data analysis of single-crystal samples has revealed that the polar direction related to the inversion symmetry breaking of $\mathrm{MoTe}_{2}$ in the low-temperature phase is along the $c$ axis [21]. The $p$-polarized SH photons with $p$-polarized incidence are recorded as the probe.The pump is also $p$ polarized. Obviously, the change of the probe is proportional to the change of the polar degree [as we demonstrate in Figs. 1(f) and 1(g)].

Figure 4(a) shows the temperature-dependent relative change of the SHG intensity at fixed pump fluence about $2 \mathrm{~mJ} / \mathrm{cm}^{2}$ as a function of the time delay. We find that the SHG intensity has no response above $T_{V}$, while it drops to a lower value quickly (about $0.7 \mathrm{ps}$ ) upon pump excitation and lasts over $100 \mathrm{ps}$ below $T_{V}$. The SH intensity in the $T_{d}$ phase drops rapidly as the temperature decreases and saturates at about $50 \mathrm{~K}$. The $0.42-\mathrm{THz}$ (period about 2 ps) phonon is also visible below $150 \mathrm{~K}$ in Fig. 4(a). Though the linear phonon spectroscopy also detects the 

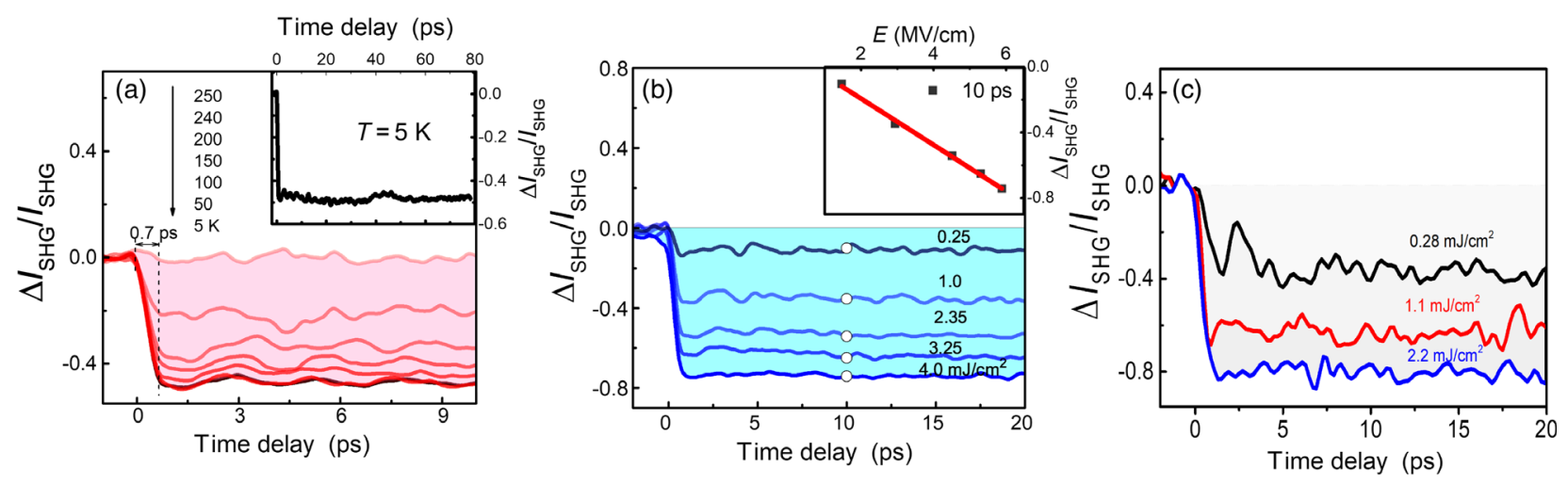

FIG. 4. (a) The relative change of SHG at fixed pump fluence of $800 \mathrm{~nm}$ about $2 \mathrm{~mJ} / \mathrm{cm}^{2}$ measured at different temperatures. The inset is the signal at longer time delay. (b) and (c) are pump-induced SHG time traces of $\mathrm{MoTe}_{2}$ at selected fluences at $T=10 \mathrm{~K}$. The inset of (b) is the relative change of SHG at various pump field strengths. The pump pulse has a wavelength of $800 \mathrm{~nm}$ with $100-\mathrm{fs}$ pulse width in (b) and a wavelength of $2.6 \mu \mathrm{m}$ with 35 -fs pulse width in (c). Both of their incident probe pulses have a wavelength of $800 \mathrm{~nm}$.

0.42-THz shear mode, the mechanisms behind the dynamical oscillation through the linear and nonlinear probes are different. The oscillation in pump-probe spectroscopy has been ascribed to the displacive excitation of the coherent phonon mechanism which is linked to Raman tensor, while the oscillation in SHG has often been taken as a hyperRaman process [17]. The symmetry of the potential changes under intense fluence at low temperature is already demonstrated from the coherent phonon spectrum in Fig. 3. The reduction of the SH signal after intense pump implies that the polar degree of the crystal indeed decreases but not vanishes yet; namely, the positions of the atoms have not yet shifted to the totally centrosymmetric phase. The outof-equilibrium SHG signal might be influenced by other effects such as the excited carriers and electron-phonon scattering mechanism, but it can be ignored since SHG is so sensible to the lattice symmetry that it is often taken as a tool to detect the symmetry change of a compound such as GaAs [42]. It is worthy to note that even at $240 \mathrm{~K}$, which is very close to the structural phase-transition temperature, the relative reduction of the SHG signal is found to be about $20 \%$. It means that the strong pump at this fluence does not drive the compound to the $T^{\prime}$ phase. On this basis, a simple laser heating effect appears to be unlikely.

To explore the impact of pump fluence on the polar of the compound, we perform fluence-dependent time-resolved SHG measurements at $T=10 \mathrm{~K}$ [Fig. 4(b)]. We can see that the shape of the SHG transient does not change, and the intensity drops with the increase of the fluence. The $\mathrm{SH}$ intensity drops to a maximum of nearly $80 \%$ but does not completely disappear at a fluence of $4 \mathrm{~mJ} / \mathrm{cm}^{2}$. This is probably because the Gaussian-like beam profile leads to a larger effect in the center of the beam than at the edge where the optical intensity is weaker, and not all of the $T_{d}$ phase enters the normal-semimetal $1 T^{\prime}$ phase. The $0.42-\mathrm{THz}$ coherent phonon disappears at higher fluence. The fast decrease of signal intensity within about $0.7 \mathrm{ps}$ after pumping suggests that the structural transition takes place in a subpicosecond timescale. This timescale is much smaller than the timescale of the increase of the lattice temperature arising from the coupling of optically excited hot electrons to the phonon modes, which could last for several hundreds of picoseconds $[12,40]$. The corresponding electric field intensity could be estimated from the equation $E_{p}=\sqrt{2 Z_{F_{0}}\left(P / A_{\text {eff }}\right)}$, where $Z_{F_{0}}=377 \Omega, A_{\text {eff }}$ is the beam size at focus, $P=\left(w / \tau_{\mathrm{FWHM}}\right), w$ is the pulse energy, and $\tau_{\text {FWHM }}$ is the duration of pulse [43]. From the inset of Fig. 4(b), we can see that the relative change of the SHG intensity scales linearly with the electric field intensity. It is likely that this phase transition is closely related to field strength.

To verify the nonthermal picture, we further perform a time-resolved SHG measurement with the pump excitation wavelength at $2.6 \mu \mathrm{m}$ being tuned from the idler beam of an optical parametric amplifier pumped by another amplified Ti:sapphire laser system at $800 \mathrm{~nm}$ with 35 -fs duration and $1-\mathrm{kHz}$ repetition rate. At such a longer wavelength, the reflectivity of the compound becomes higher, $R \approx 0.55$ due to metallic response, and the optical penetration depth becomes much longer $\delta \approx 250 \mathrm{~nm}$ based on our own reflectance measurement over broad frequencies and subsequent Kramers-Kronig transformation, which is also in agreement with the recent optical measurement on $\mathrm{MoTe}_{2}$ [44]. According to the equation used in the estimation of the lattice temperature increase in Sec. III, both effects lead to a smaller increase of lattice temperature when comparing with 800-nm excitations at the same fluence. As we show in Fig. 4(c), the SHG signal of the material drops to about $19 \%$ of its original value at about $2.4 \mathrm{~mJ} / \mathrm{cm}^{2}$, and its electric field intensity is $6.14 \mathrm{MV} / \mathrm{cm}$, which is consistent with the field strength in the above-800-nm pump with $100-\mathrm{fs}$ pulse width at a fluence of $4.5 \mathrm{~mJ} / \mathrm{cm}^{2}$. The lattice temperature is estimated to be less than $90 \mathrm{~K}$ by the heating effect equation at this fluence, which is far below the structural transition temperature. These results unambiguously support 
the scenario that the switching effect is not driven by the thermal effect but by the electric field of the laser pulses.

It is worthy to mention a closely related work on $\mathrm{WTe}_{2}$ by Sie et al. [45]. They performed an intense terahertz and midinfrared pump time-resolved TEM study as well as a time-resolved SHG measurement with a $2.1-\mu \mathrm{m}$ pump and found a very similar lattice symmetry switch from the $T_{d}$ to the $1 T^{\prime}$ phase [45]. Since the $\mathrm{WTe}_{2}$ compound is very similar to $\mathrm{MoTe}_{2}$ but does not have a structural phase transition at elevated temperature, their experiments rule out the possibility of the heating effect for the pumpinduced lattice symmetry change. Since the pump energy is low, they proposed an electric-field-driving mechanism to explain the phase transition. The applied field accelerates electrons away from the topmost valence band constituting an interlayer antibonding orbital. This kind of situation will change the coupling strength between layers. Interlayer shear movement makes the material reach a new equilibrium position, where the material changes from the $T_{d}$ phase into the $1 T^{\prime}$ phase. It seems that the same driving mechanism may also apply to the strong field pumping on $\mathrm{MoTe}_{2}$ with higher excitation energy of $800 \mathrm{~nm}(1.55 \mathrm{eV})$.

\section{CONCLUDING REMARKS}

The combined coherent phonon spectroscopy and the TR SHG study reveal unambiguously a photoinduced switch between the $T_{d}$ and $1 T^{\prime}$ phases in $\mathrm{MoTe}_{2}$. Several features deserve to be further addressed here. First, the timeresolved SHG measurement demonstrates that the sharp drop of the SHG signal is within a timescale of subpicoseconds after ultrafast excitations, indicating a nonthermal pathway for the photoinduced phase transition. This timescale is intrinsic since it is longer than the experimental time resolution. Second, both the pump-probe and the timeresolved SHG measurements reveal that the photoinduced state lasts for a very long time without showing noticeable decay [see inset of Fig. 4(a)]. This indicates that the intense photoexcitations actually drive the $T_{d}$ phase of the compound to a metastable phase, though this metastable phase is likely to be a known one in equilibrium, i.e., a monoclinic $1 T^{\prime}$ phase. In fact, for many quantum materials, ultrashort pulses can drive them to certain novel quantum phases inaccessible in a typical tuning method (temperature, chemical doping, pressure), such as bismuth [46], $1 T-\mathrm{TaS}_{2}$ [47], and $\mathrm{WTe}_{2}$ [45]. Third, it is well known that the energy difference between the $T_{d}$ and $1 T^{\prime}$ phases is small. Theoretical calculations [10] suggested that the hole doping can destroy the orthorhombic phase and stabilize the monoclinic phase in $\mathrm{MoTe}_{2}$ once the doped hole density exceeds approximately $10^{20} \mathrm{~cm}^{-3}$. Different from the hole doping, the ultrashort photoexcitation presented in this work offers a new route to manipulate different orders in $\mathrm{MoTe}_{2}$. Our experiments indicate a nonthermal lattice symmetry transition and suggest that the electric field of laser excitations plays an important role in phase transition.
It is noted that a similar ultrafast symmetry switch was found very recently in $\mathrm{WTe}_{2}$ [45].

The present study represents the first realization of an ultrafast subpicosecond switch between the $T_{d}$ phase and $1 T^{\prime}$ phase in bulk $\mathrm{MoTe}_{2}$ among all possible tuning methods. It opens up new possibilities for ultrafast manipulation of the topological properties of solids, thus, enabling potentially practical applications for a topological switch device with ultrafast excitations. Our results indicate that investigating photoinduced structural change and its decay dynamics by direct time-resolved structural probes, e.g., time-resolved x-ray diffraction, would be an interesting direction to pursue in future research.

\section{ACKNOWLEDGMENTS}

We acknowledge useful help from H. P. Wang in the data analysis and useful discussions with J. Demsar. This work is supported by the National Natural Science Foundation of China (Grants No. 11888101 and No. 11774008), the National Key Research and Development Program of China (Grants No. 2017YFA0302904, No. 2016YFA0300902, No. 2018YFA0305604, and No. 2017YFA0303302), and the Key Research Program of the Chinese Academy of Sciences (Grant No. XDPB08-2).

[1] A. A. Soluyanov, D. Gresch, Z. Wang, Q. Wu, M. Troyer, X. Dai, and B. A. Bernevig, Type-II Weyl Semimetals, Nature (London) 527, 495 (2015).

[2] Y. Sun, S.-C. Wu, M. N. Ali, C. Felser, and B. Yan, Prediction of Weyl Semimetal in Orthorhombic $\mathrm{MoTe}_{2}$, Phys. Rev. B 92, 161107(R) (2015).

[3] T. R. Chang, S. Y. Xu, G. Chang, C. C. Lee, S. M. Huang, B. K. Wang, G. Bian, H. Zheng, D. S. Sanchez, I. Belopolski, N. Alidoust, M. Neupane, A. Bansil, H. T. Jeng, H. Lin, and M.Z. Hasan, Prediction of an ArcTunable Weyl Fermion Metallic State in $\mathrm{Mo}_{x} \mathrm{~W}_{1-x} \mathrm{Te}_{2}$, Nat. Commun. 7, 10639 (2016).

[4] W. G. Dawson and D. W. Bullett, Electronic Structure and Crystallography of $\mathrm{MoTe}_{2}$ and $\mathrm{WTe}_{2}$, J. Phys. C 20, 6159 (1987).

[5] R. Clarke, E. Marseglia, and H. P. Hughes, A LowTemperature Structural Phase Transition in $\mathrm{MoTe}_{2}$, Philos. Mag. B 38, 121 (1978).

[6] Y. Wang, J. Xiao, H. Zhu, Y. Li, Y. Alsaid, K. Y. Fong, Y. Zhou, S. Wang, W. Shi, Y. Wang, A. Zettl, E. J. Reed, and X. Zhang, Structural Phase Transition in Monolayer $\mathrm{MoTe}_{2}$ Driven by Electrostatic Doping, Nature (London) 550, 487 (2017).

[7] Y. Qi et al., Superconductivity in Weyl Semimetal Candidate $\mathrm{MoTe}_{2}$, Nat. Commun. 7, 11038 (2016).

[8] H. Takahashi, T. Akiba, K. Imura, T. Shiino, K. Deguchi, N. K. Sato, H. Sakai, M. S. Bahramy, and S. Ishiwata, Anticorrelation between Polar Lattice Instability and 
Superconductivity in the Weyl Semimetal Candidate $\mathrm{MoTe}_{2}$, Phys. Rev. B 95, 100501(R) (2017).

[9] F. C. Chen, X. Luo, R. C. Xiao, W. J. Lu, B. Zhang, H. X. Yang, J. Q. Li, Q. L. Pei, D. F. Shao, R. R. Zhang, L. S. Ling, C. Y. Xi, W. H. Song, and Y. P. Sun, Superconductivity Enhancement in the s-Doped Weyl Semimetal Candidate $\mathrm{MoTe}_{2}$, Appl. Phys. Lett. 108, 162601(R) (2016).

[10] H.-J. Kim, S.-H. Kang, I. Hamada, and Y.-W. Son, Origins of the Structural Phase Transitions in $\mathrm{MoTe}_{2}$ and $\mathrm{WTe}_{2}$, Phys. Rev. B 95, 180101(R) (2017).

[11] T. Huber, S. O. Mariager, A. Ferrer, H. Schäfer, J. A. Johnson, S. Grübel, A. Lübcke, L. Huber, T. Kubacka, C. Dornes, C. Laulhe, S. Ravy, G. Ingold, P. Beaud, J. Demsar, and S. L. Johnson, Coherent Structural Dynamics of a Prototypical Charge-Density-Wave-to-Metal Transition, Phys. Rev. Lett. 113, 026401 (2014).

[12] S. Wall, D. Wegkamp, L. Foglia, K. Appavoo, J. Nag, R. F. Haglund, Jr., J. Stähler, and M. Wolf, Ultrafast Changes in Lattice Symmetry Probed by Coherent Phonons, Nat. Commun. 3, 721 (2012).

[13] P. Beaud, S. L. Johnson, E. Vorobeva, U. Staub, R. A. De Souza, C. J. Milne, Q. X. Jia, and G. Ingold, Ultrafast Structural Phase Transition Driven by Photoinduced Melting of Charge and Orbital Order, Phys. Rev. Lett. 103, 155702 (2009).

[14] P. Beaud et al., A Time-Dependent Order Parameter for Ultrafast Photoinduced Phase Transitions, Nat. Mater. 13, 923 (2014).

[15] D. Hsieh, J. W. McIver, D. H. Torchinsky, D. R. Gardner, Y.S. Lee, and N. Gedik, Nonlinear Optical Probe of Tunable Surface Electrons on a Topological Insulator, Phys. Rev. Lett. 106, 057401 (2011).

[16] A. Y. Bykov, T. V. Murzina, N. Olivier, G. A. Wurtz, and A. V. Zayats, Coherent Lattice Dynamics in Topological Insulator $\mathrm{Bi}_{2} \mathrm{Te}_{3}$ Probed with Time-Resolved Optical Second-Harmonic Generation, Phys. Rev. B 92, 064305 (2015).

[17] Y.-M. Chang, L. Xu, and H. W. K. Tom, Coherent Phonon Spectroscopy of GaAs Surfaces Using Time-Resolved Second-Harmonic Generation, Chem. Phys. 251, 283 (2000).

[18] H. J. Zeiger, J. Vidal, T. K. Cheng, E. P. Ippen, G. Dresselhaus, and M. S. Dresselhaus, Theory for Displacive Excitation of Coherent Phonons, Phys. Rev. B 45, 768 (1992).

[19] T. E. Stevens, J. Kuhl, and R. Merlin, Coherent Phonon Generation and the Two Stimulated Raman Tensors, Phys. Rev. B 65, 144304 (2002).

[20] Y. Wang, J. Xiao, H. Zhu, Y. Li, Y. Alsaid, K. Y. Fong, Y. Zhou, S. Wang, W. Shi, Y. Wang, A. Zettl, E. J. Reed, and X. Zhang, Structural Phase Transition in Monolayer $\mathrm{MoTe}_{2}$ Driven by Electrostatic Doping, Nature (London) 550, 487 (2017).

[21] H. Sakai, K. Ikeura, M. S. Bahramy, N. Ogawa, D. Hashizume, J. Fujioka, Y. Tokura, and S. Ishiwata, Critical Enhancement of Thermopower in a Chemically Tuned Polar Semimetal MoTe 2 , Sci. Adv. 2, e1601378 (2016).

[22] C. Zhang, S. Kc, Y. Nie, C. Liang, W. G. Vandenberghe, R. C. Longo, Y. Zheng, F. Kong, S. Hong, R. M. Wallace, and K. Cho, Charge Mediated Reversible Metal-Insulator Transition in Monolayer $\mathrm{MoTe}_{2}$ and $\mathrm{W}_{x} \mathrm{Mo}_{1-x} \mathrm{Te}_{2}$ Alloy, ACS Nano 10, 7370 (2016).
[23] A. Cavalleri, Cs. Tóth, C. W. Siders, J. A. Squier, F. Ráksi, P. Forget, and J.C. Kieffer, Femtosecond Structural Dynamics in $\mathrm{VO}_{2}$ during an Ultrafast Solid-Solid Phase Transition, Phys. Rev. Lett. 87, 237401 (2001).

[24] M. Rini, R. Tobey, N. Dean, J. Itatani, Y. Tomioka, Y. Tokura, R. W. Schoenlein, and A. Cavalleri, Control of the Electronic Phase of a Manganite by Mode-Selective Vibrational Excitation., Nature (London) 449, 72 (2007).

[25] A. Tomeljak, H. Schäfer, D. Städter, M. Beyer, K. Biljakovic, and J. Demsar, Dynamics of Photoinduced Charge-Density-Wave to Metal Phase Transition in $\mathrm{K}_{0.3} \mathrm{MoO}_{3}$, Phys. Rev. Lett. 102, 066404 (2009).

[26] S. Cho, S. Kim, J. H. Kim, J. Zhao, J. Seok, D. H. Keum, J. Baik, D.-H. Choe, K. J. Chang, K. Suenaga, S. W. Kim, Y.H. Lee, and H. Yang, Phase Patterning for Ohmic Homojunction Contact in $\mathrm{MoTe}_{2}$, Science 349, 625 (2015).

[27] L. Huang, T. M. McCormick, M. Ochi, Z. Zhao, M.-T. Suzuki, R. Arita, Y. Wu, D. Mou, H. Cao, J.-Q. Yan, N. Trivedi, and A. Kaminski, Spectroscopic Evidence for a Type II Weyl Semimetallic State in $\mathrm{MoTe}_{2}$, Nat. Mater. 15, 1155 (2016).

[28] K. Deng, G. Wan, P. Deng, K. Zhang, S. Ding, E. Wang, M. Yan, H. Huang, H. Zhang, Z. Xu, J. Denlinger, A. Fedorov, H. Yang, W. Duan, H. Yao, Y. Wu, S. Fan, H. Zhang, X. Chen, and S. Zhou, Experimental Observation of Topological Fermi Arcs in Type-II Weyl Semimetal MoTe ${ }_{2}$, Nat. Phys. 12, 1105 (2016).

[29] Y. M. Dai, J. Bowlan, H. Li, H. Miao, S. F. Wu, W. D. Kong, P. Richard, Y. G. Shi, S. A. Trugman, J.-X. Zhu, H. Ding, A. J. Taylor, D. A. Yarotski, and R. P. Prasankumar, Ultrafast Carrier Dynamics in the Large-Magnetoresistance Material $\mathrm{WTe}_{2}$, Phys. Rev. B 92, 161104(R) (2015).

[30] Q. Wang, Y. Ji, S. Ge, X. Li, J. Qiu, J. Feng, and D. Sun, Valley Carrier Dynamics in Mono Layer Molybdenum Disulfide from Helicity-Resolved Ultrafast Pump-Probe Spectroscopy, ACS Nano 7, 11087 (2013).

[31] K. Zhang, C. Bao, Q. Gu, X. Ren, H. Zhang, K. Deng, Y. $\mathrm{Wu}$, Y. Li, J. Feng, and S. Zhou, Raman Signatures of Inversion Symmetry Breaking and Structural Phase Transition in Type-II Weyl Semimetal $\mathrm{MoTe}_{2}$, Nat. Commun. 7, 13552 (2016).

[32] S.-Y. Chen, T. Goldstein, D. Venkataraman, A. Ramasubramaniam, and J. Yan, Activation of New Raman Modes by Inversion Symmetry Breaking in Type II Weyl Semimetal Candidate (T)-MoTe 2 , Nano Lett. 16, 5852 (2016).

[33] R. Beams, L. G. Cançado, S. Krylyuk, I. Kalish, B. Kalanyan, A. K. Singh, K. Choudhary, A. Bruma, P. M. Vora, F. Tavazza, A. V. Davydov, and S. J. Stranick, Characterization of Few-Layer $1 T \mathrm{MoTe}_{2}$ by PolarizationResolved Second Harmonic Generation and Raman Scattering, ACS Nano 10, 9626 (2016).

[34] Y. Zhao, X. Luo, H. Li, J. Zhang, P. T. Araujo, C. K. Gan, J. Wu, H. Zhang, S. Y. Quek, M. S. Dresselhaus, and Q. Xiong, Interlayer Breathing and Shear Modes in FewTrilayer $\mathrm{MoS}_{2}$ and $\mathrm{WSe}_{2}$, Nano Lett. 13, 1007 (2013).

[35] M. Hase, K. Mizoguchi, H. Harima, S. Nakashima, M. Tani, K. Sakai, and M. Hangyo, Optical Control of Coherent Optical Phonons in Bismuth Films, Appl. Phys. Lett. 69, 2474 (1996). 
[36] B. J. Siwick, V. R. Morrison, R. P. Chatelain, K. L. Tiwari, A. Hendaoui, A. Bruhács, and M. Chaker, A Photoinduced Metal-Like Phase of Monoclinic $\mathrm{VO}_{2}$ Revealed by Ultrafast Electron Diffraction, Science 346, 445 (2014).

[37] N. Gedik, D.-s. Yang, G. Logvenov, I. Bozovic, and A. H. Zewail, Nonequilibrium Phase Transitions in Cuprates Observed by Ultrafast Electron Crystallography, Science 316, 425 (2007).

[38] J. W. Harter, D. M. Kennes, H. Chu, A. de la Torre, Z. Y. Zhao, J.-Q. Yan, D. G. Mandrus, A. J. Millis, and D. Hsieh, Evidence of an Improper Displacive Phase Transition in $\mathrm{Cd}_{2} \mathrm{Re}_{2} \mathrm{O}_{7}$ via Time-Resolved Coherent Phonon Spectroscopy, Phys. Rev. Lett. 120, 047601 (2018).

[39] B. He, C. Zhang, W. Zhu, Y. Li, S. Liu, X. Zhu, X. Wu, X. Wang, H.-h. Wen, and M. Xiao, Coherent Optical Phonon Oscillation and Possible Electronic Softening in $\mathrm{WTe}_{2}$ Crystals, Sci. Rep. 6, 30487 (2016).

[40] A. Cavalleri, Cs. Tóth, C. W. Siders, J. A. Squier, F. Ráksi, P. Forget, and J. C. Kieffer, Femtosecond Structural Dynamics in $\mathrm{VO}_{2}$ during an Ultrafast Solid-Solid Phase Transition, Phys. Rev. Lett. 87, 237401 (2001).

[41] M. Fiebig, V. Pavlov, and R. V. Pisarev, Second-Harmonic Generation as a Tool for Studying Electronic and Magnetic
Structures of Crystals: Review, J. Opt. Soc. Am. B 22, 96 (2005).

[42] P. Saeta, J. K. Wang, Y. Siegal, N. Bloembergen, and E. Mazur, Ultrafast Electronic Disordering during Femtosecond Laser Melting of GaAs, Phys. Rev. Lett. 67, 1023 (1991).

[43] F. Blanchard, L. Razzari, H.-C. Bandulet, G. Sharma, R. Morandotti, J.-C. Kieffer, T. Ozaki, M. Reid, H. F. Tiedje, H. K. Haugen, and F. A. Hegmann, Generation of $1.5 \mu \mathrm{J}$ Single-Cycle Terahertz Pulses by Optical Rectification from a Large Aperture ZnTe Crystal, Opt. Express 15, 13212 (2007).

[44] S.-i. Kimura, Y. Nakajima, Z. Mita, R. Higashinaka, T. D. Matsuda, and Y. Aoki, Optical Evidence of Type-II Weyl Semimetals $\mathrm{MoTe}_{2}$ and $\mathrm{WTe}_{2}$, arXiv:1902.09888v1.

[45] E. J. Sie et al., An Ultrafast Symmetry Switch in a Weyl Semimetal, Nature (London) 565, 61 (2019).

[46] D. M. Fritz and J. B. Hastings, Ultrafast Bond Softening in Bismuth: Mapping a Solid's Interatomic Potential with X-Rays, Science 315, 633 (2007).

[47] L. Stojchevska, I. Vaskivskyi, T. Mertelj, P. Kusar, D. Svetin, S. Brazovskii, and D. Mihailovic, Ultrafast Switching to a Stable Hidden Quantum State in an Electronic Crystal, Science 344, 177 (2014). 